\title{
Oral mucositis as a pathway for fatal outcome among critically ill patients exposed to chlorhexidine: post hoc analysis of a randomized clinical trial
}

\author{
Wanessa Teixeira Bellissimo-Rodrigues ${ }^{1}$, Mayra Gonçalves Menegueti ${ }^{2}$, Leandro Dorigan de Macedo ${ }^{3}$,
} Anibal Basile-Filho ${ }^{4}$, Roberto Martinez ${ }^{5}$ and Fernando Bellissimo-Rodrigues ${ }^{1,2^{*}}$ (D)

Keywords: Chlorhexidine, Mucositis, Mortality, Respiratory tract infections, Dental care, Critical care

Chlorhexidine (CHX) oral application has been widely used for preventing respiratory infections among critically ill patients, despite controversial effectiveness and the suspicion that it could enhance their mortality [1-3]. The physiopathology behind this association is poorly understood [2, 3]. Our objective was to reassess data from a clinical trial searching for potential pathways for the CHXassociated mortality [4, 5].

This is a post hoc analysis of a randomized clinical trial evaluating a dental care intervention aimed to prevent respiratory infections in the intensive care unit (ICU) setting. Adult patients admitted to the study ICU between January 1, 2011, and August 8, 2013, were eligible if they had a perspective of staying for 2 days. Participants were randomized by the dentist using a dice. The experimental group received dental care provided by a dentist plus routine oral care, while the control group had access only to routine oral care provided by the nursing staff. Both groups used $0.12 \% \mathrm{CHX}$ oral solution, if

\footnotetext{
* Correspondence: fbellissimo@usp.br

${ }^{1}$ Social Medicine Department, Ribeirão Preto Medical School, University of São Paulo, Campus Universitário, s/n, Monte Alegre, Ribeirão Preto, São Paulo 14048-900, Brazil

${ }^{2}$ Infection Control Service, University Hospital of Ribeirão Preto Medical

School, University of São Paulo, Ribeirão Preto, Brazil

Full list of author information is available at the end of the article
}

fully conscious, or $2 \% \mathrm{CHX}$ oral gel, if unconscious, three times a day throughout their ICU stay.

Adverse events potentially related to oral care procedures were pragmatically assessed at least three times a week in both study groups by the dentist during ICU stay. Their relationship with death in the ICU was evaluated through a logistic regression model, adjusting the outcome for sex, age, and Acute Physiology and Chronic Health Evaluation System II (APACHE II) score. Sample size was calculated based on the primary study outcome and the lower respiratory tract infection incidence and pointed to the inclusion of 294 patients.

Study "per protocol" population consisted of 254 patients and $9.84 \%(25 / 254)$ of them had adverse events related to oral care procedures, being the most common CHX-induced mucositis (7.09\%, 18/ 254 ), consisting of oral erosive or ulcerative lesions, along with white plaque formation [6]. Only one patient had previous lesions before exposure to CHX. This adverse event was exclusively reported in patients exposed to $2 \%$ CHX oral gel $(9.28 \%, 18 / 194$, $p=0.006)$ and was found to be associated with a fatal outcome in both univariate and multivariate analysis, as shown in Table 1.

Most of the patients who died had infection and sepsis as their direct cause of death $(56 / 77,72.7 \%)$. Table 2 describes the occurrence of $\mathrm{CHX}$-induced mucositis and its association with direct causes of death and temporal outcomes reported during ICU stay. 
Table 1 Clinical and demographic characteristics of patients and occurrence of adverse events related to oral care procedures evaluated as potential risk factors for death during ICU stay

\begin{tabular}{llllll}
\hline Clinical and demographic characteristics & Discharged alive & Death in the ICU & Crude RR (95\%Cl) & Adjusted OR (95\%Cl) & Adjusted $p$ value \\
\hline Female & $69.4(84 / 121)$ & $30.6(37 / 121)$ & - & - & - \\
Male & $69.9(93 / 133)$ & $30.1(40 / 133)$ & $0.98(0.68-1.43)$ & $0.83(0.45-1.52)$ & 0.553 \\
Age in years & $57(41-71)$ & $62(52-71)$ & $1.02(1.00-1.03)$ & $1.00(0.98-1.02)$ & 0.781 \\
APACHE II score & $20(16-26)$ & $27(21-31)$ & $1.10(1.06-1.15)$ & $1.10(1.05-1.15)$ & $<0.001$ \\
Routine oral care & $68.5(87 / 127)$ & $31.5(40 / 127)$ & - & - & $0.92(0.50-1.67)$ \\
Dental treatment & $70.9(90 / 127)$ & $29.1(37 / 127)$ & $0.92(0.64-1.34)$ & 0.779 \\
Without adverse events related to oral care & $73.4(168 / 229)$ & $26.6(61 / 229)$ & - & - & - \\
With any adverse event related to oral care & $36.0(9 / 25)$ & $64.0(16 / 25)$ & $2.40(1.67-3.46)$ & $5.46(2.11-14.13)$ & $<0.001$ \\
Without CHX-induced mucositis & $72.5(171 / 236)$ & $27.5(65 / 236)$ & - & - & - \\
With CHX-induced mucositis & $33.3(6 / 18)$ & $66.7(12 / 18)$ & $2.42(1.64-3.56)$ & $6.14(1.98-19.08)$ & 0.002 \\
Without intraoral bleeding & $71(174 / 245)$ & $29(71 / 245)$ & - & - & - \\
With intraoral bleeding & $33.3(3 / 9)$ & $66.7(6 / 9)$ & $2.30(1.40-3.80)$ & $3.74(0.75-18.58)$ & 0.106 \\
\hline
\end{tabular}

Values expressed are \% $(\mathrm{n} / \mathrm{N})$ of patients for categorical variables and median (interquartile range) for continuous variables

ICU intensive care unit, APACHE II Acute Physiology and Chronic Health Evaluation System II, RR relative risk, OR odds ratio

In the present study, we could not assess whether CHX application enhanced or not the mortality of the studied patients because all of them were exposed to it. However, examining the adverse events potentially related to oral care procedures, we found the CHX-induced mucositis was strongly and independently associated with death, even when the association was adjusted for sex, age, and the patients' baseline severity of illness score. Consistently, patients affected by $\mathrm{CHX}$-induced mucositis had a prolonged length of stay in the ICU and mechanical ventilation and were submitted to longer periods of antimicrobial therapy. Of great concern is the fact that the interruption of the $2 \% \mathrm{CHX}$ oral gel application after identification of mucositis did not prevent these patients to clinically deteriorate, eventually leading $2 / 3(12 / 18)$ of them to die in the ICU.

In conclusion, our data points to oral mucositis as the main pathway for the association between CHX exposure and enhanced in-hospital mortality. The disruption of the oral mucosa integrity possibly leads to the translocation of bacteria from the oral cavity to the bloodstream, therefore enhancing the likelihood of infection and sepsis. In our opinion, the use of oral CHX among hospitalized patients should be strictly restricted to those with established intraoral infections, such as periodontal disease, preferentially applied by a dentist.

Table 2 Occurrence of CHX-induced mucositis and its association with direct causes of death and temporal outcomes reported during ICU stay

\begin{tabular}{|c|c|c|c|}
\hline Outcome & Without mucositis \% (n/N) & With CHX-induced mucositis \% (n/N) & RR $(95 \% \mathrm{Cl})$ \\
\hline Death, in general & $27.5(65 / 236)$ & $66.7(12 / 18)$ & $2.42(1.64-3.56)$ \\
\hline Death due to any infection & $20.3(48 / 236)$ & $44.4(8 / 18)$ & $2.18(1.23-3.88)$ \\
\hline Death due to respiratory infection & $10.6(25 / 236)$ & $22.2(4 / 18)$ & $2.10(0.82-5.37)$ \\
\hline Death due to intrabdominal infection & $5.93(14 / 236)$ & $16.7(3 / 18)$ & $2.81(0.89-8.88)$ \\
\hline Death due to acute respiratory failure & $3.4(8 / 236)$ & $5.6(1 / 18)$ & $1.64(0.22-12.39)$ \\
\hline Death due to cardiovascular events & $2.5(6 / 236)$ & $0(0 / 18)$ & 0 \\
\hline Temporal outcome & $\begin{array}{l}\text { Without CHX-induced mucositis } \\
\text { Median (interquartile range) }\end{array}$ & $\begin{array}{l}\text { With CHX-induced mucositis } \\
\text { Median (interquartile range) }\end{array}$ & $p$ value $^{a}$ \\
\hline Duration of mechanical ventilation (days) & $7(3-16)$ & $13(8-20)$ & 0.023 \\
\hline Duration of antimicrobial therapy (days) & $5(2-11)$ & $12.5(8-18)$ & 0.002 \\
\hline Length of stay in the ICU (days) & $7(4-15)$ & $14(9-20)$ & 0.003 \\
\hline
\end{tabular}




\section{Acknowledgements}

The authors thank the Intensive Care Team and the Infection Control Service of the study facility for supporting the study implementation.

\section{Authors' contributions}

WTBR conceived the study, performed the literature review, collected most of the data, and wrote the first version of the manuscript. MGM collected some of the study outcomes, participated in the analysis of the data, and revised the final version of the manuscript. LDM participated in the analysis of the data and preparation and revision of the manuscript. ABF and RM participated in the planning and implementation of the study, analysis of the data, and revision of the final manuscript. FBR conceived the study and participated in all stages of its implementation, including planning, collecting data, analyzing data, and preparing and revising the manuscript. All authors read and approved the final manuscript.

\section{Funding}

The clinical trial was supported by Fundação de Amparo à Pesquisa do Estado de São Paulo (FAPESP), process no. 2010/51063-4, and Fundação de Apoio ao Ensino, Pesquisa e Assistência do Hospital das Clínicas da Faculdade de Medicina de Ribeirão Preto da Universidade de São Paulo (FAEPA), both non-profit organizations.

\section{Availability of data and materials}

The datasets used and/or analyzed during the current study are available from the corresponding author on reasonable request.

\section{Ethics approval and consent to participate}

The study protocol was submitted and approved by institutional (Comitê de Ética em Pesquisa do Hospital das Clínicas da Faculdade de Medicina de Ribeirão Preto) and national ethics review committees before implementation. The protocol number was CAAE - 0490.0.004.000-09. Written consent was obtained from all participating patients, or from their relatives, in case of unconscious patients.

\section{Consent for publication}

Not applicable.

\section{Competing interests}

The authors declare that they have no competing interests.

\section{Author details}

${ }^{1}$ Social Medicine Department, Ribeirão Preto Medical School, University of São Paulo, Campus Universitário, s/n, Monte Alegre, Ribeirão Preto, São Paulo 14048-900, Brazil. ${ }^{2}$ Infection Control Service, University Hospital of Ribeirão Preto Medical School, University of São Paulo, Ribeirão Preto, Brazil. ${ }^{3}$ Dental Service, University Hospital of Ribeirão Preto Medical School, University of São Paulo, Ribeirão Preto, Brazil. ${ }^{4}$ Intensive Care Medicine Division, Department of Surgery and Anatomy, Ribeirão Preto Medical School, University of São Paulo, Ribeirão Preto, Brazil. ${ }^{5}$ Infectious Diseases Division, Internal Medicine Department, Ribeirão Preto Medical School, University of São Paulo, Ribeirão Preto, Brazil.

Received: 21 October 2019 Accepted: 4 November 2019

Published online: 27 November 2019

\section{References}

1. Klompas M, Speck K, Howell MD, Greene LR, Berenholtz SM. Reappraisal of routine oral care with chlorhexidine gluconate for patients receiving mechanical ventilation: systematic review and meta-analysis. JAMA Intern Med. 2014;174:751-61.

2. Price R, MacLennan G, Glen J, SuDDICU Collaboration. Selective digestive or oropharyngeal decontamination and topical oropharyngeal chlorhexidine for prevention of death in general intensive care: systematic review and network meta-analysis. BMJ. 2014;348:g2197.

3. Deschepper M, Waegeman W, Eeckloo K, Vogelaers D, Blot S. Effects of chlorhexidine gluconate oral care on hospital mortality: a hospital-wide, observational cohort study. Intensive Care Med. 2018;44:1017-26.

4. Bellissimo-Rodrigues WT, Menegueti MG, Gaspar GG, Nicolini EA, Auxiliadora-Martins M, Basile-Filho A, et al. Effectiveness of a dental care intervention in the prevention of lower respiratory tract nosocomial infections among intensive care patients: a randomized clinical trial. Infect Control Hosp Epidemiol. 2014;35:1342-8.

5. Bellissimo-Rodrigues WT, Menegueti MG, Gaspar GG, de Souza HCC, Auxiliadora-Martins M, Basile-Filho A, et al. Is it necessary to have a dentist within an intensive care unit team? Report of a randomised clinical trial. Int Dent J. 2018;68:420-7.

6. Plantinga NL, Wittekamp BHJ, Leleu K, Depuydt P, Van den Abeele A-M, Brun-Buisson C, et al. Oral mucosal adverse events with chlorhexidine $2 \%$ mouthwash in ICU. Intensive Care Med. 2016:42:620-1.

\section{Publisher's Note}

Springer Nature remains neutral with regard to jurisdictional claims in published maps and institutional affiliations.
Ready to submit your research? Choose BMC and benefit from:

- fast, convenient online submission

- thorough peer review by experienced researchers in your field

- rapid publication on acceptance

- support for research data, including large and complex data types

- gold Open Access which fosters wider collaboration and increased citations

- maximum visibility for your research: over $100 \mathrm{M}$ website views per year

At BMC, research is always in progress.

Learn more biomedcentral.com/submissions 